\title{
DIFERENCIAS SECTORIALES DE SALARIOS ENTRE TRABAJADORES PRIVADOS URBANOS EN URUGUAY*
}

\author{
Marisa Bucheli**
}

\section{Resumo}

Este trabalho tem como objetivo fazer uma estimativa das diferenças salariais entre setores de atividade no Uruguai (1988), considerando aspectos regionais e características individuais na estimaçāo. As estimativas foram feit as para o total dos homens do setor privado urbano e para as diferentes ocupaçōes, achando-se estruturas salariais semelhantes entre elas que sugerem que a diferenciaçāo salarial nāo pode ser atribuida apenas às características de of erta ou regionais. Embora, as mulheres apresentam uma estrutura diferente sug erindo a existência de uma segmentação baseada no sexo.

Palavras chave: Diferenças salariais, distribuiçāo de salários, estrutura de salărios

\section{Abstract}

The objective of this paper is to estimate sectorial wage differences for Uruguay (1988), controlling for individual characteristics and geographical region. The estimations were made for all the men of the private urban sector and for different occupational groups. The wage structure doesn't diff er substantially accross those groups, but women present a differente one: segmentation between men and women seems to be important.

Ke y words: Sectorial wage differentials, earnings distribution, inter-industry wage structure

\section{Introducción.}

Existe un conjunto de literatura,empírica que reporta la existencia de diferencias salariales estables entre trabajadores de mismas características, que desempeñan actividades aparentemente sinzilares, pero que se insertan en diferentes sectores de actividad.

Ello ha contribuido a apuntalar la idea de que existe una segmentación en el mercado de trabajo como resultado de las diferencias - basadas en aspectos tecnológicos - entre sectores productivos.

*El presente artículo se sustenta en la tesis de maestrado en economia realizada por la autora (PUC-RJ).

**A sistente de investigación en el Instituto de Economia (F.C.E., Uruguay).

R. de Econometria Rio de Janeiro v. X, no 2, p.337-355 novembro 1990 
Un sector que concentra empresas con características como alta rentabilidade, grande tamaño, cierto grado de monopolio en su mercado de bienes, baja participación del trabajo en los costos, alta relación capital-trabajo, tiene capacidad de pagar salarios superiores a sectores de características opuestas. Se ha encontrado que estas características están fuertemente correlacionadas entre ellas y con el grado de sindicalización (particularmente lo está el grado de concentración en el mercado de bienes), el cual mide la capacidad de los trabajadores de hacer efectivo el pago de salarios relativamente altos.

Criterios basados en un salario de eficiencia (una productividad endógena que aumenta con el salario justifica niveles salariales por encima de los de equilibrio, que serán mayores cuánto más altos los costos de rotación y selección, mayor la dificultad de supervisión del personal, etc.) justifican además la necesidad de pagar salarios altos por parte de las empresas. En tanto el salario de eficiencia varie entre sectores, se sustentan los diferenciales de salarios.

Existe cierta evidencia de que la estructura de salarios industriales en Uruguay se ha mantenido estable entre 1980 y 1085 tanto como para obreros como para empleados ${ }^{1}$. El coeficiente de variación del salario medio de los obreros ha sido $31.6 \%$ en 1080 . $26.8 \%$ en 1982 y $30.3 \%$ en 1985 ; el del salario medio de los empleados ha sido $27.2 \%$ en $1980,26.4 \%$ en 1982 y $31.5 \%$ en 1985 . Estos resultados fueron obtenidos sin control de las características de los trabajadores, utilizando el salario medio de cada rama industrial clasificadas a cuatro dígitos.

El objeto de este trabajo es medir las diferencias de salarios (por hora) entre sectores de producción en Uruguay teniendo en cuenta las características individuales que se espera que influyan en el salario de los trabajadores. De esta forma se intenta evitar el sesgo que se ocasiona cuando algún sector concentra trabajadores de determinado sexo, calificación o región. Para eso se ha utilizado la información de la Encuesta de Hogares que permite trabajar con datos individuales sobre salario, sector de inserción, ocupación desempeñada, edad, educación, sexo y zona geográfica. Otra de las ventajas de la Encuesta es que la información no se limita a la industria, aunque existe el inconveniente de que la

${ }^{1}$ Bucheli-Rossi (1988) 
máxima apertura de la clasificación sectorial es de dos dígitos. A continuación, en el punto 2 , se realiza una reseña de los datos con que se ha trabajado.

En el punto 3 se presentan los resultados obtenidos. Una primera estimación fue realizada para el conjunto de los hombres del sector privado, encontrando que los servicios y el comercio pagan salarios inferiores al transporte y construcción, seguidos de la industria, mientras que el sistema bancario se despega como el pagador de los mayores salarios. Posteriormente se realizaron estimaciones para los hombres de las distintas ocupaciones y finalmente para las mujeres.

\section{Los datos.}

Los datos utilizados provienen de la Encuesta INacional de Hogares realizada por la Dirección General de Estadistica y Censos de Uruguay (año 1988), la que se refiere únicamente a la población u rbana. Se ha trabajado con hombres y mujeres por separado, tomando los trabajadores ocupados en el sector privado excepto los que trabajan en establecimientos agropecuarios, los militares y los p rofesionales. Luego de eliminar estos casos más otros en que no existían o no podían estimarse las características individuales o el salario, quedaron 6570 hombres y 4616 mujeres.

Las características individuales controladas son la educación, experiencia, zona geográfica y ocupación.

A cada individuo se la asignó con EDUC (educación) el número de años lectivos que cursó en instituciones formales de enseñanza. La variable experiencia está aproximada por EXP, calculada como la edad menos los años lectivos cursados menos 5, por ser 5 el número de años en que no se recibe educación formal. EXPC es el cuadrado de la variable EXP y EXPED es el producto de EDC y EXP.

La zona geográfica se ha controlado por medio de una variable dummy DREG que vale 1 para los trabajadores de Montevideo y 0 para los del resto del país.

En la muestra se cuenta con información sobre la ocupación de los trabajadores con una clasificación a tres dígitos. Pero existen problemas para su utilización cuando se quiere trabajar con sectores de producción, ya que la codificación de estos últimos está estrechamente relacionada a la clasificación de ocupaciones, 
impidiendo el uso de ambas informaciones en regresiones econométricas. De todas formas, la utilización de la clasificación a tres dígitos no parece importante ya que la existencia de distintas ocupaciones en ese nivel responde a los requerimientos de los propios sectores.

Se ha trabajado entonces con seis grandes grupos ocupacionales a efectos de un control, ya que su existencia no depende de la presencia de un sector particular y existen además entre ellos seis, claras diferencias en las características necesarias para su desempeño, sea por el diferente grado de intensidad del trabajo manual o porque corresponden a diferentes posiciones en la escala de mandos.

La ocupación entonces se ha controlado con seis variables dummy: DTSP toma valor 1 para los trabajadores en servicios personales y ocupaciones afines; DTCD para los gerentes, administradores y funcionarios de categoria directiva; DEMP para los empleados de oficina y personas en ocupaciones afines; DVEN para los comerciantes, vendedores y afines; DCON para los conductores de medios de transporte y afines; DOBR para los obreros $\mathrm{y}$ artesanos.

Los sectores se han identificado con variables dummy DRA Mi que toman valor 1 cuando el individuo trabaja en el sector $i$. La información de la Encuesta está abierta según la Clasificación Internacional Industrial Uniforme (CIIU) a dos dígitos, y se ha trabajado con esta codificación y con una que reagrupa los sectores a un dígito. El total de variables y su definición son los siguientes:

DRAM2: Explotación de minas y canteras.

DRAM3: Industrias manufactureras.

DRAM31: Productos alimenticios, bebidas y tabaco.

DRAM32: Textiles, prendas de vestir e industrias del cuero.

DRAM33: Industria y productos de la madera.

DRAM34: Fabricación de papel y productos del papel, imprentas y editoriales.

DRAM35: Fabricación de sustancias químicas y de productos químicos, derivados del petróleo y del carbón, de caucho y plástico. DRAM36: Fabricación de productos minerales no metálicos, excepto los derivados del petróleo y carbón.

DRAM37: Industrias metálicas básicas. 
DRAM38: Fabricación de productos metálicos, maquinaria y equipo.

DRAM39: Otras industrias manufactureras.

DRAM4: Electricidad, gas y agua.

DRAM5: Construcción.

DRAM6: Comercio.

DRAM61: Comercio al por mayor.

DRAM62: Comercio al por menor.

DRAM63: Cafés, bares, restoranes y hoteles.

DRAM7: Transporte y comunicaciones.

DRAM8: Banca, finanzas y servicios a empresas.

DRA M81: Instituciones financieras.

DRAM82: Seguros.

DRAM83: Servicios a empresas.

DRAM9: Servicios comunales, sociales y personales.

DRAM91: Servicios de la administración central.

DRAM92: Servicios de saneamiento y afines.

DRAM93: Instituciones de enseñanza y servicios médicos.

DRAM94: Servicios de esparcimiento.

DRAM95: Talleres de reparaciones, mantenimiento, limpieza y afines.

DRAM96: Otros servicios.

Obsérvese que en la clasificación a dos dígitos se mantienen agregados los setores $2,4,5$ y 7 . Los tres primeros no se encuentran sub-clasificados en la encuesta y el sector 7 agrupa dos ramas, siendo que una contiene solamente seis casos: se ha optado entonces por mantenerlo a un dígito.

La información sobre ingresos corresponde al mes anterior al que la persona fue encuestada. Asi, los datos abarcan información correspondiente al período diciembre de 1987 - noviembre de 1988.

Se ha trabajado con el salario (excluido horas extras, beneficios sociales, aguinaldo, salario vacacional, ingresos en especie y otras gratificaciones) percibido por la ocupación principal dividido por las horas trabajadas (excepto las extras) en esa ocupación. Un inconveniente es que las horas trabajadas se refieren a las de la semana anterior, lo que obliga a multiplicar por cuatro y trabajar con un total de horas que no tiene por qué coincidir con las del mes anterior, que es al que se refiere el salario. Por eso cuando 
notoriamente existió una discrepancia entre las informaciones (en particular salarios y horas igual 0) el individuo fue eliminado.

Los salarios nominales de la encuesta no son comparables puesto que corresponden a diferentes meses. Para obtener la variable SAL, el salario por hora de la ocupación principal ha sido deflactado por el índice medio de salarios privados. El índice escogido tiene el inconveniente de no permitir sustraer de cada salario la diferencia del aumento salarial sectorial con respecto a la media durante ese año. ¿Qué tipo de error puede adjudicarse a la utilización de este indice?

En 1988 existian convenios salariales sectoriales ${ }^{2}$ que no abarcaron a todos los trabajadores y que rigieron los aumentos realizados en febrero, junio y octubre. La pauta básica es similar para todos los convenios ya que la mayoria de las correcciones deseadas por los sindicatos para equiparación de salarios de algunos grupos de trabajadores fueron conseguidas a partir de 1985 (primer año de libre actuación sindical) y previa la firma de los convenios. Si todos los trabajadores hubieran firmado convenios, sería de esperar que el error al utilizar el salario medio privado como deflactor fuera poco importante. Pero aquellos que no firmaron convenios debían regirse por los aumentos decretados por el Poder Ejecutivo $^{3}$, que fueron en las tres ocasiones inferiores a los de la pauta común negociada en los convenios.

Esto significa que el índice medio de salarios utilizado, además de estar afectado por nuevas contrataciones y otros aspectos que hacen que crezca mensualmente y no sólo los meses de aumento, presenta un crecimiento menor para quienes firmaron convenios y mayor para quienes no lo hicieron.

\section{Las diferencias salariales.}

La especificación que se ha utilizado para medir las diferencias

\footnotetext{
${ }^{2}$ La clasificación de sindicatos y cámaras firmantes no corresponde estrictamente a la CIIU lo que no permite deflactar el salario de cada trabajador por el aumento que le correspondió por convenio.

${ }^{3}$ Es de pensar que efectivamente así suce dió ya que la no firma de convenio indica que el sindicato no est aba suficientemente organizado como para hacerlo, por lo que difícilmente consiguiera organizarse en la fecha correspondiente al ajuste para obtener un aumento mayor.
} 
de salarios ha sido:

$$
\begin{aligned}
& \operatorname{lnSAL}=\alpha+\beta_{1} \mathrm{EDUC}+\beta_{2} \mathrm{EXP}+\beta_{3} \mathrm{EXPC}+\beta_{4} \mathrm{EXPED} \\
& +\beta_{5} \mathrm{DREG}+\beta_{6} \mathrm{DOCUP}+\delta \mathrm{DRAM}+u
\end{aligned}
$$

donde DOCUP se compone de las variables dummy correspondientes al grupo ocupacional y DRAM de las dummy sectoriales.

Si las variables dummy sectoriales no estuvieran correlacionadas con las demás, dos regresiones - una que explique el salario únicamente por el sector de inser ción y otra que lo haga exclusivamente por las caracteristicas individuales - permitirian realizar una descomposición de la contribución de cada conjunto de variables a la varianza explicada.

Dado que es probable que exista multicolinealidad entre las dummies sectoriales y las caracteristicas individuales, existe un efecto conjunto de los dos grupos de variables. Adjudicando este efecto conjunto a alguna de las dos fuentes de dispersión (caracteristicas individuales o sectores) puede estimarse un intervalo de la contribución del sector de actividad a la varianza explicada por la regresión.

La varianza explicada en la estimación que incluye solamente las dummies sectoriales contiene la explicación que aporta el efecto conjunto del sector y las características individuales, constituyendo un valor máximo de la contribución del sector de producción a la varianza de salarios. Asi, el valor máximo de la contribución se estimó con el $R^{2}$ de una regresión cuyas variables ex.plicativas son únicamente las dummies correspondientes a los sectores.

El valor minimo de la contribución de las variables dummy a la varianza explicada puede medirse atribuyendo el efecto conjunto de los sectores y características individuales a estas últimas. Esto es, se estimó como el aumento del poder explicativo (incremento del $R^{2}$ ) al introducir en una regresión que contiene como variables explicativas únicamente las caracteristicas individuales, un vector de dummies correspondientes a los sectores de actividad.

Se han realizado estimaciones utilizando la clasificación de sectores de producción a uno y dos dígitos. Con la clasificación a un dígito, el sector de actividad contribuye a la explicación entre 1.9 y $5.3 \%$. Con la desagregación a dos dígitos, la contribución es de entre 3.6 y $20.5 \%$. 


\section{Cuadro 1.}

Diferencias salariales de los sectores en relación al comercio (ramas 6 y 62) (\%)

\begin{tabular}{|c|c|}
\hline Clasificación a & \\
\hline sector & $\%$ \\
\hline 3 & 29.7 \\
\hline 5 & 19.8 \\
\hline 7 & 8.0 \\
\hline 8 & 49.7 \\
\hline 9 & -0.8 \\
\hline
\end{tabular}

Clasificación a dos dígitos

\begin{tabular}{rrrr} 
sector & $\%$ & sector & \multicolumn{1}{c}{$\%$} \\
31 & 35.0 & 61 & 19.4 \\
32 & 37.1 & 63 & 3.9 \\
33 & 5.9 & 7 & 16.9 \\
34 & 44.7 & 81 & 114.9 \\
35 & 60.5 & 83 & 21.1 \\
36 & 7.4 & 91 & 29.1 \\
38 & 26.6 & 93 & 19.1 \\
39 & -0.6 & 94 & -2.4 \\
5 & 20.8 & 95 & 4.7
\end{tabular}

En el cuadro no 1 se presenta la diferencia salarial de los distintos sectores en relación al comercio en la clasificación a un dígito y en relación al comercio al por menor en la desagregación a dos digitos. Se excluyeron los sectores que contienen menos de diez casos.

Para medir las diferencias salariales se estimó:

$$
\ln \mathrm{SAL}=\alpha+\beta \mathrm{CI}+\gamma_{1} \mathrm{DRAM}_{1}+\cdots+\gamma_{n-1} \mathrm{DRAM}_{n-1}+u
$$

donde ya se mencionó que DRAMi $(i=1, \ldots, n)$ es una variable dummy que vale 1 si el individuo trabaja en el sector $i$ y 0 si lo hace en nn sector $j \neq i$ y CI es el vector de caracteristicas individuales.

Si $E(u)=0$ el logaritmo del salario medio del sector $n$ puede ser estimado a través de una estimación de $\alpha$. A su vez, cada $\boldsymbol{\gamma}_{i}$ 
representa la diferencia de los logaritmos del salario de $n$ y del de $i$. La diferencia porcentual se estima operando con los $\gamma_{i}$.

Para realizar la estimación de los parámetros $\gamma_{i}$, se ha trabajado con el método de mínimos cuadrados ordinarios. El estadístico $t$ asociado a cada parámetro permite rechazar o no la hipótesis de que la diferencia salarial del sector $i$ con respecto al sector excluído es igual 0.

En el cuadro no 2, aparecen los distintos sectores ordenados según el salario medio pagado (de menor a mayor). Cuando la diferencia salarial entre el grupo-fila y el grupo-columna es distinta de 0 al $99 \%$, se colocó un asterisco en el casillero correspondiente; cuando esa diferencia es significativa al $95 \%$ se colocaron dos asteriscos.

Tanto en la estimación a un dígito como en la de dos, parece haber franjas salariales, escapándose a este comportamiento el sector de servicios a la administración central (91) y otras industrias (39).

En la agregación sectorial a un digito, se observa que el conjunto de servicios y comercio (ramas 9 y 6 ) paga los salarios menores, seguido del conjunto transporte-construcción (ramas 7 y 5). De la información de la desagregación a dos dígitos, se desprende que dentro del primer conjunto, la enseñanza y salud (93) $y$ el comercio al por mayor (61) pagan salarios significativamente mayores a los de los demás sub-sectores de ese conjunto y similares a los del transporte y construcción, insertándose en un grupo de salarios intermedios. Los salarios industriales son en su conjunto significativamente superiores a los de las ramas agregadas citadas y por último, el sector 8 es el que paga las mayores remuneraciones.

La desagregación a dos dígitos muestra que el comportamiento entre las ramas industriales es diferente. La industria de la madera (33) y minerales no metálicos (36) se encuentran entre el grupo pagador de menores salarios; la pioducción de maquinaria y equipo (38) en el grupo de salarios intermedios; salarios mayores son pagados por la industria de alimentos (31), textiles (32), pa pel (34) y productos químicos (35) en ese orden. Con respecto al sector 8 , los bancos y casas bancarias (81) son los que explican los altos salarios medios del conjunto, mientras que los servicios a empresas (83) se sitúan en la franja intermedia.

Los resultados no son sorprendentes de acuerdo a lo previsto 


\section{Cuadro 2.}

Nivel de significación de las

diferencias salariales entre sectores de produccción

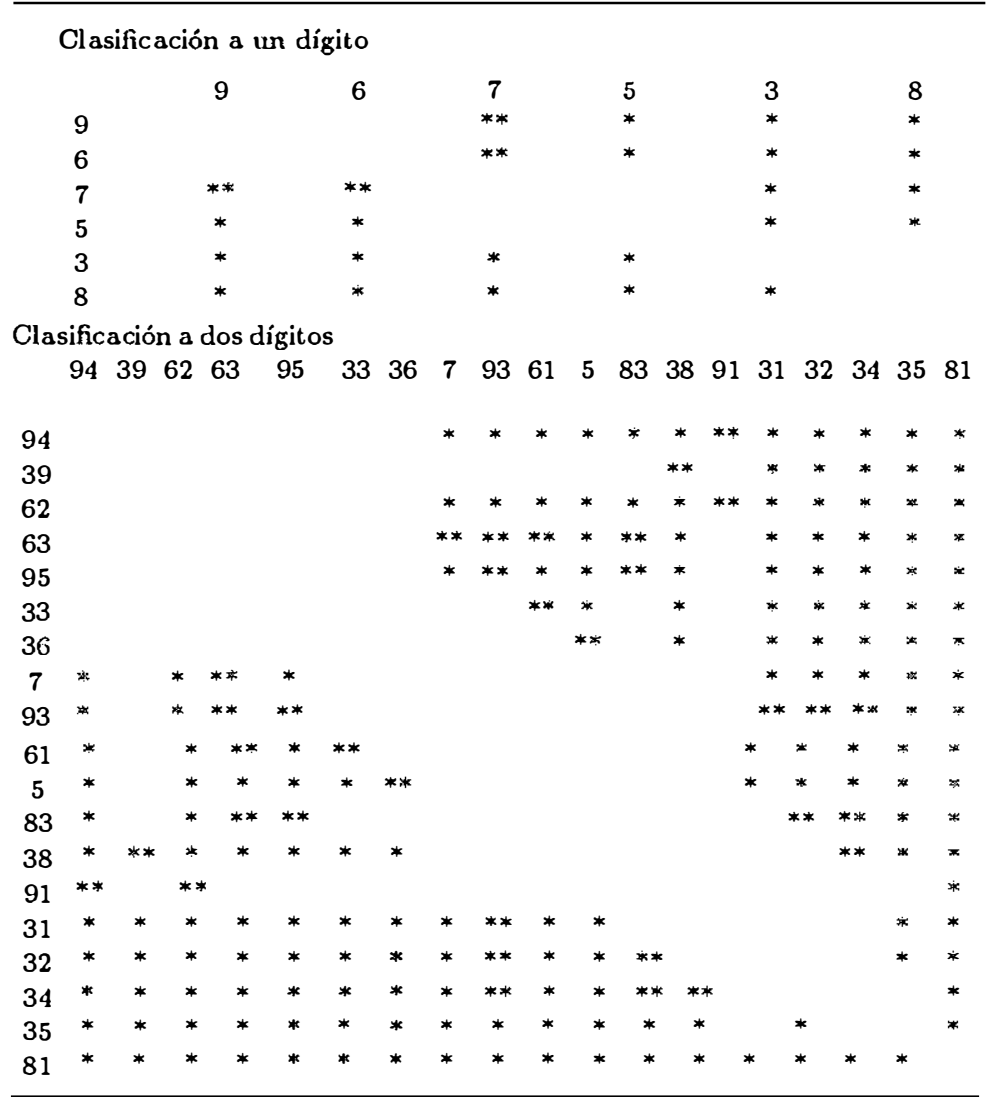

en la literatura teórica sobre segmentación. El comercio al por menor y los servicios de bares y restoranes, de esparcimiento, de reparaciones, limpieza, etc. concentran varias empresas pequeñas y de baja relación capital-trabajo., y existe un escaso o nulo grado de sindicalización. Si se compara con resultados obtenidos para Estados Unidos (Krueger y Summers, 1987) estas actividades forman parte también de las peores remuneradas en ese país, sucediendo también que los servicios de salud, enseñanza y el comercio 
al por mayor se encuentran en una mejor posición relativa al resto de los servicios. En niveles de salarios más altos se encuentran la mayoria de las ramas industriales. Dos diferencias saltan a la vista en la comparación: la posición relativa alta en Uruguay de la industria textil y de los bancos.

\section{Los salarios dentro de cada ocupación}

La clasificación de ocupaciones utilizada fue: los trabajadores de categoría directiva (TCD), empleados (EMP), vendedores y comerciantes (VEN), conductores de medios de transporte (CON), obreros y artesanos (OBR) y trabajadores de servicios personales (TSP).

Existe una concentración de los OBR en la industria y construcción, de los VEN en el comercio, de los CON en el transporte y de los TSP en los sectores servicio y comercio, mientras que los EMP y TCD están distribuidos más uniformemente entre los sectores. Esto dificulta el aislamiento del efecto del sector de producción y justifica una estimación para cada grupo ocupacional.

Algunas estimaciones pueden ayudar a entender la relación grupo ocupacional - - sector. Se realizaron tres estimaciones para aislar el efecto ocupación: una en que no aparece la indicencia del sector de producción y otras dos en que si se han tenido en cuenta en su clasificación a uno y dos digitos.

Los TSP reciben un salario significativamente menor (diferente al $99 \%$ ) a los trabajadores de las demás ocupaciones en las tres estimaciones. Medida con respecto al salario de los OBR, los TSP perciben un salario inferior en un $27 \%$ cuando se estima sin sectores. Al incluírlos, la diferencia salarial de los TSP y OBR cae a $19 \%$ (el porcentaje es similar utilizando la clasificación de uno y dos dígitos): el sector servicios y comercio estarian recogiendo parte del efecto conjunto de estar insertos en ellos y ser TSP.

Los VEN, OBR y CON reciben salarios en una franja intermedia cuando se realizan las estimaciones que incluyen los sectores de producción, no pudiendo rechazar que los salarios medios de cada ocupación sean diferentes entre ellos al $95 \%$. Pero tanto los CON como los VEN perciben salarios significativamente menores que los OBR (estimados en 7 y $8 \%$ respectivamente, no siendo estos porcentajes diferentes entre sí al $95 \%$ en la estimación sin sectores). Un efecto similar al observado con los TSP estaría sucediendo en este caso. 


\section{Cuadro 3.}

\section{Contribución a la explicación de los sectores de producción para cada grupo}

\begin{tabular}{ccc} 
Clasificación a un dígito & & \\
Grupo ocupacional & Limite inferior & Limite superior \\
TCD & 10.4 & 20.3 \\
EMP & 4.0 & 9.0 \\
VEN & 1.3 & 6.4 \\
OBR & 1.9 & 2.0 \\
CON & 0.9 & 2.5 \\
TSP & 6.4 & 6.9 \\
& & \\
Clasificación a dos dígitos & & \\
Grupo ocupacional & Limite inferior & Limite superior \\
EMP & 10.4 & 23.1 \\
OBR & 2.5 & 5.5 \\
TSP & 8.3 & 9.1 \\
\hline
\end{tabular}

Por último, en las tres estimaciones los trabajadores de los grupos EMP y TCD reciben salarios significativamente superiores (en ese orden) a los de las demás ocupaciones y diferentes entie ellos, sin que parezcan estar mayormente afectados por la inclusión de sectores. El salario medio de los EMP es $14.3 \%$ superior al de los OBR en la estimación sin sectores y $13.8 \%$ en la que incluye la clasificación a dos dígitos. Para los TCD, esos porcentajes son 95.1 y 101.8 respectivamente.

Se han realizado entonces para cada grupo ocupacional, tres estimaciones similares a las presentadas para el conjunto de los hombres. En el cuadro no 3 se presentan los limites inferiores y superiores de la contribución de los sectores de producción a la explicación de la varianza de salarios para cada grupo ocupacional.

La especificación de la regresión estimada para cada grupo es la misma que la presentada en el punto 3 , solo que sin el vector de dummies ocupacionales. Dado el número de trabajadores en cada ocupación, solo fue posible trabajar con la clasificación sectorial a dos digitos para los empleados, obreros y trabajadores en servicios personales.

En el cuadro n으 4 se presenta la diferencia salarial para cada 
grupo ocupacional de cada sector con respecto al comercio en la clasiflcación a un dígito y con respecto al sub-sector comercio al por menor en la desagregación a dos dígitos. Obsérvese que no coinciden todos los sectores que aparecen en los cuadros para las distintas ocupaciones: es que nuevamente se han eliminado de la presentación las ramas con menos de diez casos, y ellas son diferentes para cada ocupación.

En general, con la apertura a un digito puede decirse que existe coincidencia entre los sectores pagadores de bajos, medianos y altos salarios para los distintos grupos ocupacionales, aunque para los vendedores y conductores de medios de transporte - con quienes solo fue posible trabajar con la clasificación a un dígito - los resultados fueron poco importantes en términos de la significación de las diferencias.

Más interesante es observar los tres grupos para los cuales fue posible trabajar a dos dígitos. Al igual que para el conjunto de los hombres, para las tres distintas ocupaciones los servicios y el comercio aparecen como pagadores de bajos salarios, excepto la enseñanza y salud y el comercio al por mayor (excepto para los TSP) que presentan salarios medios mayores al resto del conjunto.

La industria paga salarios superiores a los del servicio y comercio para todas las ocupaciones, excepto los sub-sectores de productos minerales no metálicos (36) y de la madera (33) que aparecen solamente para los OBR. Y quienes pagan los salarios medios más altos para todas las ocupaciones son las instituciones financieras (81).

Existen disparidades e u el comportamiento de la construcción y el transporte. Ambos sectores aparecen como de bajos salarios para los TSP, lo es la construcción para los EMP pero el transporte es de franja intermedia, y ambos son de franja intermedia para los OBR.

La existencia de coincidencias en el comportamiento de los sectores entre grupos ocupacionales es importante. Si se observa por ejemplo la rama 93 para los TSP, se ve que ella paga salarios superiores al resto de los servicios. Las explicaciones tradicionales dirian que ese diferencial compensa determinadas condiciones de trabajo desventajosas o que no ha sido posible observar determinadas habilidades específicas de este sub-grupo de trabajadores. Sin embargo la rama 93 paga salarios medios superiores también a los obreros y empleados: parecería mucha casualidad que las con- 
Cuadro 4.

Diferencia salarial de los sectores con respecto al comercio (ramas 6 y 62) por grupo ocupacional (\%)

$\begin{array}{crrrrrrr}\text { Clasificación a un digito } & & & & & & \\ \text { sector } & & \text { EMP } & \text { CON } & \text { VEN } & \text { TSP } & \text { TCD } & \text { OBR } \\ & 3 & 29.6 & 11.9 & 22.2 & 38.6 & 18.6 & 21.9 \\ 5 & 31.7 & 65.0 & & -11.7 & & 11.5 \\ 7 & -5.6 & 6.0 & & 15.8 & & 21.8 \\ 8 & 98.5 & & 49.4 & 82.8 & 53.4 & 44.9 \\ 9 & 2.3 & -5.9 & & -2.1 & -43.5 & 8.1\end{array}$

Clasificación a dos digitos

$\begin{array}{lrrrr}\text { sector } & \text { EMP } & \text { TSP } & \text { OBR } \\ 31 & 66.1 & 75.2 & 34.1 & \\ 32 & 21.8 & 47.8 & 41.1 \\ 33 & & & 8.4 \\ 34 & 49.9 & 58.2 & 54.4 \\ 35 & 99.8 & & 4.5 \\ 36 & & & 32.7 \\ 38 & 7.3 & & -6.8 \\ 39 & & 1.1 & 20.2 \\ 5 & 47.4 & 5.9 & 14.3 \\ 61 & 31.1 & 18.8 & 8.5 \\ 63 & -8.6 & 32.3 & 31.8 \\ 7 & 3.8 & 156.6 & \\ 81 & 116.0 & & \\ 83 & 11.3 & 62.3 & 72.5 \\ 93 & 29.3 & 17.0 & 5.4 \\ 94 & -9.3 & 2.1 & 14.8 \\ 95 & -6.2 & & \end{array}$

diciones de trabajo o un error de medición operaran en el mismo sentido para los tres grupos ocupacionales. Las observaciones de coinciden cias tienden más bien a reafirmar que existe una incidencia propia del sector sobre los salarios.

\section{Los salarios de las mujeres}

¿Existirán también coincidencias entre la estructura salarial de hombres y mujeres? Pareceria que no, pero algunas presiciones sobre la inser ción de las mujeres ayudan a comprender las diferen- 


\section{Cuadro 5. \\ Diferencias salariales de los sectores \\ en relación al comercio (ramas 6 y 62) (\%)}

$\begin{array}{cr}\text { Clasificación a un digito } & \\ \text { sector } & \% \\ 3 & 3.8 \\ 5 & 31.2 \\ 7 & 19.2 \\ 8 & 2.8 \\ 9 & -7.4\end{array}$

Clasificación a dos dígitos

\begin{tabular}{cccc} 
sector & $\%$ & sector & $\%$ \\
31 & 13.3 & 61 & 16.9 \\
32 & -10.3 & 63 & -0.2 \\
33 & -2.3 & 7 & 28.6 \\
34 & 13.6 & 81 & 29.4 \\
35 & 22.6 & 83 & 3.8 \\
36 & 10.9 & 93 & 21.8 \\
38 & 38.6 & 94 & -2.0 \\
39 & 14.4 & 95 & -22.3 \\
\hline
\end{tabular}

cias encontradas entre las estructuras salariales. En particular, se cuenta con muy pocos casos en algunos sectores.

En la clasificación a un dígito, como con los hombres el conjunto servicios-comercio paga un salario significativamente menor. Pero los otros cuatro sectores no presentan diferencias significativas entre ellos aunque llama la atención que el transporte (7) y construcción (5) paguen salarios superiores a la industria (3) y sector financiero (8).

La observación de la inserción de hombres y mujeres en los sectores puede ayudar a explicar estos resultados.

La proporción de mujeres que trabajan en la construcción y el transporte es bien inferior a la de hombres. En particular son administrativas, y para esa ocupación estos dos sectores presentan de las menores relaciones mujeres en el total de trabajadores del sector. Sectores que contratan pocas mujeres, quizá decidan hacerlo cuando ellas presentan importantes habilidades que no son observables y por lo tanto no controlables.

Eliminando entonces la construcción y el transporte, queda 
por saber por qué el sector 8 no aparece despegado del resto como con los hombres. Recuérdese que de la clasificación a dos digitos para los hombres se desprende que este comportamiento lo lidera el sub-sector 81 (bancos), y mientras que el $50 \%$ de los hombres del sector 8 trabaja en particular en el 81 , eso es válido solamente para el $30 \%$ de las mujeres. Eso influye en un salario medio del sector 8 inferior en las mujeres, pero de la clasificación a dos dígitos surge que como con los hombres, los bancos (81) también pagan salarios de la franja superior a las mujeres.

La clasificación a dos digitos muestra también algunos comportamientos dispares entre hombres y mujeres, en particular cuando se observa los niveles de significación (Cuadro $n$ 으 6).

Los servicios de reparaciones, mantenimiento y afines (95), que absorben al $42.6 \%$ de las mujeres, pagan el salario más bajo y diferente del de todos los demás sectores al $99 \%$, cosa que no sucedia con los hombres. Una probable explicación es que hombres y mujeres se inserten en renglones diferentes dentro del sector, no pudiendo cuantificarse por la falta de desagregación sectorial a más digitos.

Aparece luego de la rama 95, un conjunto de sectores pagadores de salarios no significativamente diferentes entre elos. Hay aqui otra discrepancia con respecto a los resultados obtenidos para los hombres: la aparición de la industria textil entre este grupo, que si se elimina la rama 95 es el pagador de salarios bajos.

Mientras, el grupo pagador de mayores salarios se compone (además de los ya mencionados transporte, instituciones financieras y construcción) de la enseñanza y salud (93), fabricación de productos químicos (35) y de maquinaria y equipo (38). Nótese que para los hombres, de las tres ramas mencionadas solamente la 35 aparecia dentro las pagadoras de salarios más altos. Nuevamente la rama 36 contrata muy pocas mujeres (ellas son principalmente obreras y representan el $7 \%$ del total de obreros contratados en el sector) por lo que vale una observación similar a la del transporte y construcción.

En sintesis, las estructuras salariales de hombres y mujeres parecen presentar discrepancias relevantes que responden más bien a inserciones diferentes en el mercado de trabajo.

\section{Conclusiones.}

La medición de las diferencias salariales entre los trabaja- 


\author{
Cuadro 6.
}

Nivel de significación de las

diferencias salariales entre sectores de producción

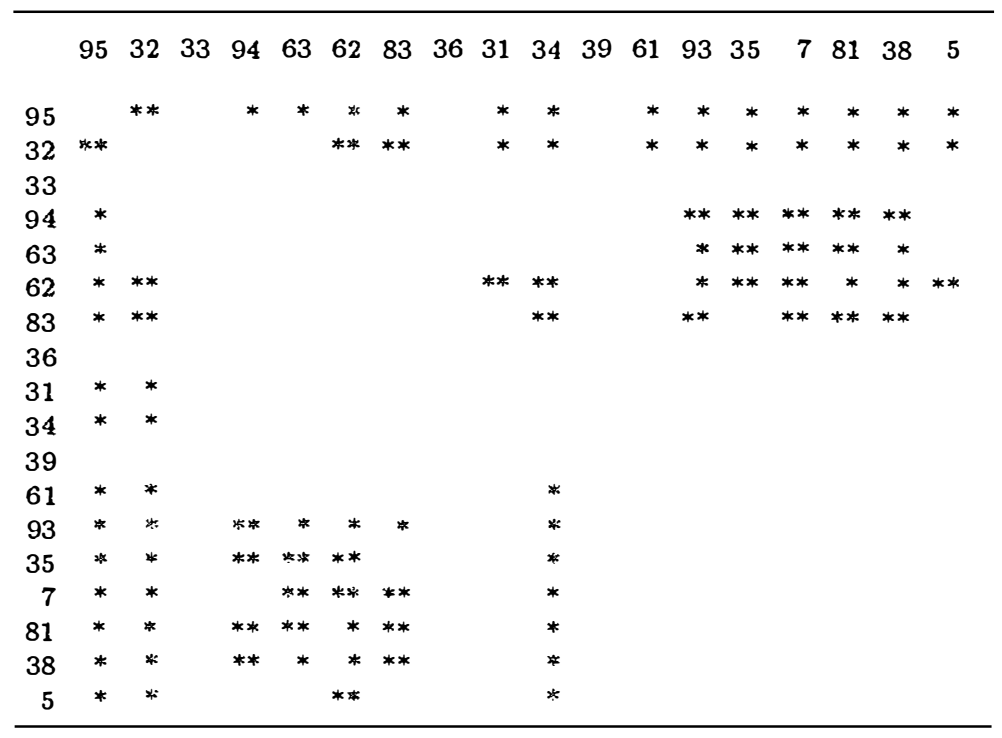

dores hombres del sector privado ha permitido detectar franjas de salarios: la menor, pagada en los servicios y el comercio, una intermedia que nuclea transporte y construcción seguida por la industria y por-último el sistema finarciero. Existe además cierta dispersión entre los salarios pagados dentro de los servicios, comercio, industria y finanzas que la clasificación a dos dígitos detecta, incluso con diferencias significativamente distintas de 0 entre las ramas de un sector. Con esta clasificación se observa una contribución del $3.6 \%$ al $10.5 \%$ del sector de actividad a la explicación de la varianza de salarios.

Las estimaciones para las diferentes ocupaciones sugieren estructuras salariales similares, en particular cuando se utilizó la clasificación a dos dígitos. Ello reafirma la idea de indicencia propia del sector en la formación del salario, de forma que cada uno respeta los salarios relativos de los trabajadores de distintas ocupaciones.

Por último, las estimaciones realizadas para las mujeres re- 
sultaron diferentes de las de los hombres. La diferente inserción de hombres y mujeres en los distintos sectores puede explicar esa diferencia. Particularmente, en el caso de sectores con bajo porcentaje de mujeres puede existir una segregación tal que los empleadores contraten personas del sexo femenino cuándo estas presentan cualidades muy por en cima de la media. Si estas no son observables, las variables de educación y experien cia no han conseguido ejer cer el control necesario. De todas formas, cualquiera sean las explicaciones sobre la diferencia de estructuras salariales entre hombres y mujeres, parece claro que la segrnentación en el mercado de trabajo basada en el sexo es relevante.

\section{Referências}

Akerloff, G. Gift exchange and efficiency wage theory: four views. American Economic Review, Papers and Proceedings, 74, no3:79-83, May 1984.

Bucheli, M. \& Rossi, M. La estructura de los salarios industriales. Summa, 4: 105-128, Abril 1988.

Cain, G. C. The challenge of segmented labor market theories to orthodox theory: a survey. Journal of Economic Literature, 1215-1248.

Dickens, W.T. \& Lang, K. The reemergence of segmented labor market theory. The American Economic Review, Papers and Proceedings, 78, n으 2:129-133, May 1988.

Gatica, J., Mizala, A. y Romaguera, P. Estructura salarial y diferenciales de salario en la industria de transformación brasilera. Proyecto PNUD/OIT/BRA/87/002.

Harrison, B. \& Sum, A. The theory of "dual" or segmented labor markets. Journal of Economic Issues, XIII, n으 3:687-706, Set. 1979.

Krueger, A.B. \& Summers, L.H. Reflections on the inter-industry wage structure. In Kevin Lang y Jonathan. S. Leonard (eds), Unemployment and the structure of labor markets pp.17-47. 1987.

Efficiency wages and the inter-industry wage

structure. Econometrica, 56, no 2:259-293, March 1988.

Savedoff, W.D. Regional wage differences and segmentation in

Brazil's urban labor markets. IPEA discussion paper, mimeo. 
Yellen, J.. Efficiency Wages Models of Unemployment. 
